Ferenc Tóth

\title{
Un milieu culturel centre-européen en Lorraine: l'Académie du roi Stanislas à Nancy et les nobles hongrois à l'époque des Lumières
}

L'Académie de Stanislas de Nancy est l'une des académies les plus prestigieuses de France, possédant un passé riche et multiculturel et qui, bénéficiant de l'apport de diverses influences, a constitué en même temps un pôle intellectuel européen depuis sa création au juste milieu du siècle des Lumières (1750). Durant mes recherches doctorales et ultérieures, j'ai beaucoup travaillé sur l'immigration hongroise en France au XVIII ${ }^{\mathrm{e}}$ siècle, et j'ai constaté une concentration signifiante de Hongrois dans les provinces limitrophes de l'Empire (Alsace et Lorraine), qui bénéficiait également du soutien de l'ancien roi de Pologne, Stanislas Leszczynski, depuis 1737 grand-duc de Lorraine, bienfaiteur et fondateur de plusieurs institutions dont la célèbre Académie. Le gouvernement de Stanislas représentait une époque de réformes particulièrement riche pour la Lorraine. Plusieurs établissements d'inspiration éclairée furent fondés. Parmi ceux-ci, la bibliothèque fondée en 1750 à Nancy, devenue plus tard une académie célèbre de grande influence sous le nom de Société royale des Sciences et Belles-Lettres de Nancy (aujourd'hui l'Académie de Stanislas) attira un grand nombre d'intellectuels à cette époque. ${ }^{1}$ L'influence de cette Académie était sûrement considérable sur les nobles hongrois attachés au service du roi Stanislas. Ayant travaillé sur la production littéraire des différents membres de la communauté hongroise en France, je me propose d'examiner une éventuelle influence du rayonnement intellectuel de la cour de Stanislas sur les nobles hongrois installés à proximité de cette nouvelle institution. Dans cette étude, je présente les relations personnelles qui existaient entre certains nobles hongrois et les milieux intellectuels lorrains, avant de montrer les étapes de la fondation de l'Académie de Stanislas. In fine, je résume l'activité littéraire de quelques nobles hongrois lettrés liés à la cour de Stanislas et leurs éventuelles relations avec le réseau académique.

1 Voir sur le sujet: Jean-Claude Bonnefont (dir.): Stanislas et son Académie. Nancy 2003. 


\section{Stanislas Leszczynski et les Hongrois en Lorraine}

La Lorraine fut l'une des régions européennes les plus controversées dans l'histoire. A la fin du XVII ${ }^{\mathrm{e}}$ siècle, elle connut une période de troubles et d'affaiblissement de la puissance ducale. Le duc Charles $\mathrm{V}$ de Lorraine ne fut pas reconnu par la France qui occupait les duchés à cette époque. Après 1697, la Lorraine retrouva son indépendance sous le règne du fils du duc Charles V, Léopold $\mathrm{I}^{\mathrm{er}}$, qui entreprit de restaurer ses États. Afin de signaler la souveraineté de son règne, il fit construire le château-résidence de Lunéville et destina son fils aîné, le duc François III de Lorraine, à une alliance impériale. Le mariage du duc François avec Marie-Thérèse d'Autriche, considéré comme le " Mariage du siècle » créa une tension politique en Europe du fait de la position géographique de la Lorraine. ${ }^{2}$ Finalement, la question de la Lorraine fut étroitement liée à celle de la succession de la Pologne, le véritable enjeu pour la France au cours de la guerre de Succession de Pologne étant la possession des duchés de Lorraine et de Bar. En faisant abstraction du corps de secours français envoyé en Pologne pour le roi Stanislas, beau-père de Louis XV, les opérations militaires de l'armée française se limitaient aux fronts occidentaux. Après la guerre, le roi Stanislas s'installa en Lorraine. Il y fonda une petite cour dont le comte Ladislas Berchény, son grand écuyer, fut un des dignitaires les plus importants (Ill. 1). ${ }^{3}$ Il en résulta l'apparition d'une petite communauté hongroise dans l'entourage du roi Stanislas. Mais d'où venait cette amitié envers les Hongrois?

Pour la bonne compréhension des choses, il convient de rappeler les relations entre Hongrois et Polonais au début du XVIII ${ }^{\mathrm{e}}$ siècle. Cette période correspondait en Hongrie à la guerre d'indépendance (1703-1711) menée par le prince François II Rákóczi contre les Habsbourg. Le prince Rákóczi, conformément à la politique de ses ancêtres, entretenait de bonnes relations avec la Pologne où il pouvait s'appuyer sur plusieurs familles aristocratiques, comme les Potocki, les Lubomirski, les Sieniawski ou les Leszczynski. En 1701, le prince Rákóczi et le comte Nicolas Berchényi trouvèrent refuge en Pologne où ils se rallièrent aux familles soutenant l'alliance avec la Suède, en particulier avec Stanislas Leszczynski, qui fut bientôt élu et couronné roi de Pologne pour la première fois. Suite à la bataille de Poltava (1709), les fragments de l'armée de

2 Voir sur ce mariage dynastique: Renate Zedinger: Hochzeit im Brennpunkt der Mächte. Franz Stephan von Lothringen und Erzherzogin Maria Theresia. Wien, Graz 1994.

3 József Zachar: Franciaország magyar marsallja, Bercsényi László [Ladislas Bercsényi, maréchal de France hongrois]. Budapest 1987, p. 126. 


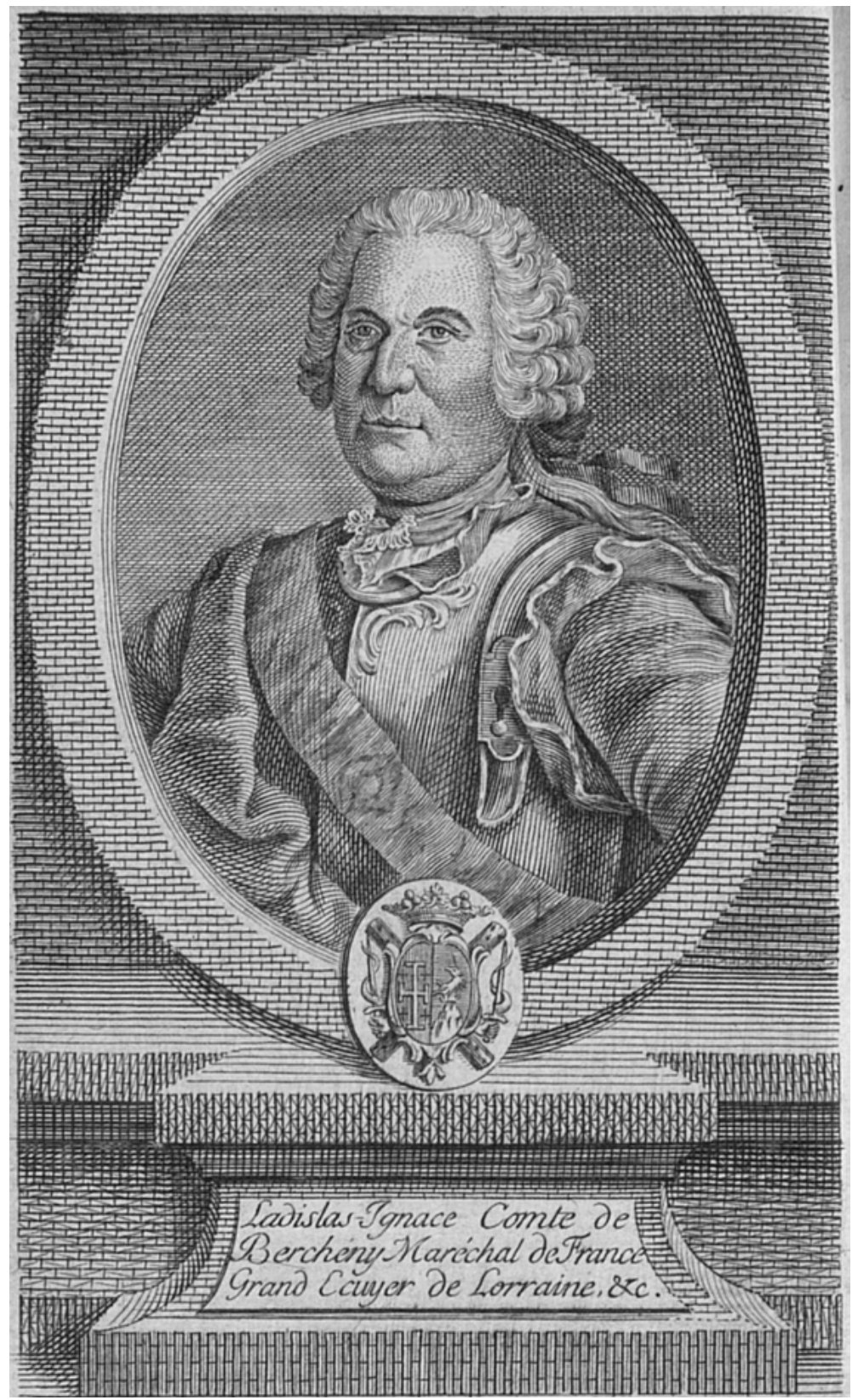

Illustration 1: Le portrait du comte de Berchény dans Le politique vertueux (Nancy, 1762) de François-Hubert Aubert (Bibliothèques de Nancy). 
Leszczynski rejoignirent celles de Rákóczi où ils furent employés comme troupes auxiliaires. ${ }^{4}$ Après la défaite de la guerre d'indépendance hongroise, Rákóczi dut quitter son pays. Le prince se réfugia d'abord avec son entourage dans le sud de la Pologne, puis il décida de s'installer à Dantzig. Vers la fin de l'année 1712, le prince partit pour la France d'où il passa en Turquie, où il termina sa vie en 1735 dans la ville de Rodosto. De même, ses officiers et partisans le suivirent dans son émigration en France. Beaucoup d'anciens combattants de la guerre d'indépendance trouvèrent un emploi au sein de l'armée royale française où ils furent intégrés dans les fameux régiments de hussards. ${ }^{5}$

Lorsque le roi Stanislas s'installa en 1719 à Wissembourg en Alsace, il habita non loin de Haguenau, le lieu de stationnement du régiment de hussards du comte Ladislas Berchény levé en 1720. Là, une véritable amitié se noua entre les deux hommes exilés, dont nous connaissons assez bien les détails grâce à une correspondance du comte Berchényi qui se trouve actuellement à la Bibliothèque Nationale Széchényi de Budapest. ${ }^{6}$ La correspondance des deux personnages commence par une lettre du roi polonais exilé, datée du 5 août 1722, dans laquelle il recommande au comte Berchény l'un de ses compatriotes pour servir dans son nouveau régiment de hussards. ${ }^{7}$ Ensuite, la correspondance devient plus amicale et plus intime, car il s'agit non seulement d'échanges de cadeaux, mais aussi d'informations secrètes. Quelques années plus tard, la politique orientale de la France favorisa les bonnes relations franco-polonaises, les émigrés hongrois en devenant également bénéficiaires. En 1725, le mariage de Louis XV avec la fille du roi Stanislas Leszczynski changea considérablement la situation du roi exilé. ${ }^{8}$ Il quitta bientôt Wissembourg et s'installa d'abord à Strasbourg,

4 En cette qualité, le contingent polonais participa à la bataille de Romhány, le 22 janvier 1710. Grâce aux travaux récents de Tamás Oláh, nous connaissons les parties de ce contingent polonais dont les régiments de garde du corps de Stanislas Leszczynski faisaient partie intégrante. Tamás Oláh: Zemplén vármegye és térsége hadi krónikája 1710 elején [La région et l'histoire militaire du comitat de Zemplén au début de 1710]. In: István Czigány, Katalin Mária Kincses (éd.): Az újrakezdés esélye. Tanulmányok a Rákóczi-szabadságharc befejezésének 300. évfordulója alkalmából. Budapest 2012, pp. 58-61.

5 Voir sur ce sujet: József Zachar: Idegen hadakban [Dans des armées étrangères]. Budapest 1981.

6 Országos Széchényi Könyvtár, Kézirattár (Bibliothèque Nationale Széchényi, section des manuscrits, dorénavant OSZKK), série Quart. Gall. 39 Lettres du roy de Pologne et d'autres princes

7 Malgré le fait qu'un régiment polonais, le régiment Royal-Pologne, existait depuis 1653 en France, les soldats polonais servaient volontiers dans les régiments de hussards ce qui prouve la bonne relation entre les deux communautés émigrées. Cf. Lydia Scher-Zembitska: Les Polonais au service de France de 1732 à 1832. Thèse de doctorat sous la direction de M. A. Corvisier. Université Paris-Sorbonne (Paris IV) 1993, pp. 305-309.

8 Michel Antoine: Louis XV, Paris 1989, p. 157. 
ensuite au château de Chambord mis à sa disposition par Louis XV. L'éloignement des deux personnes ne fit que renforcer leur amitié, ce dont témoigne leur correspondance de plus en plus affectionnée dans laquelle les plaisirs de la famille prennent une place importante. En 1725, Berchény épousa une demoiselle alsacienne et acheta des propriétés foncières en Brie. ${ }^{9}$ L'amour et le mariage du comte Berchény furent applaudis et fêtés par Leszczynski qui salua la dulcinée

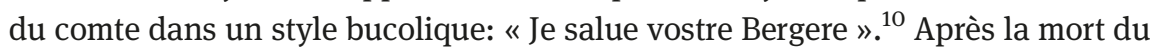
père de Berchény, Stanislas invita ainsi le comte chez lui afin de soulager ses dépenses: « Soyez sage, ne mangez pas votre argent à Paris, revenez à Chambord, car je crois que voilà un séjour qui vous consumera une partie de vos bons ducats. Je vous attends avec impatience. $~^{11}$

Stanislas Leszczynski s’informa par l'intermédiaire du comte Berchény sur les événements politiques en Europe centrale et orientale. Les agents du comte parcoururent les régions frontalières de l'Empire ottoman pour recruter des hussards hongrois et disposèrent ainsi d'un réseau de communication étendu. Par ailleurs, la diplomatie française employait volontiers des agents issus des émigrations hongroises. Certains furent chargés de missions particulières, d'autres, sous couvert d'un poste diplomatique comme celui de consul de France en Crimée, avaient des missions politiques secrètes concernant la Hongrie. Parmi eux, on relève des noms de la guerre d'indépendance hongroise tels que ceux d'Adam Jávorka, Adam Máriássy, Ladislas Berchény, André Tóth, et d'une manière indirecte, le secrétaire francophile du prince Rákóczi, Clément Mikes. ${ }^{12}$ La politique de la France envers les Malcontents hongrois s'intégrait dans cette ligne d'alliance de revers et par conséquent était considérée, surtout au XVIII ${ }^{\mathrm{e}}$ siècle, comme une affaire liée à celles de l'Empire ottoman, base de ce système d'alliance antihabsbourgeois. De cette manière, la diplomatie française pouvait éveiller les sentiments de liberté des Hongrois réfugiés en Turquie lorsqu'elle en avait besoin. Ce moyen fut particulièrement favorisé par la diplomatie secrète des rois de France, le fameux «Secret du Roi ». C'était une diplomatie parallèle dont les objectifs

9 Gyula Forster: Utóhang gróf Berchényi László, Franciaország magyar marsallja történetéhez [Contribution à l'histoire du comte Ladislas Berchényi, maréchal de France hongrois]. Budapest 1929, pp. 8-9.

10 OSZKK, série Quart. Gall. 39 Lettres du roy de Pologne et d'autres princes fol. 6 .

11 Idem. Cité par le Général Raymond Boissau: Ladislas Bercheny Magnat de Hongrie, Maréchal de France. Paris, Budapest, Szeged 2006, p. 45.

12 Sur Mikes, voir récemment: Kelemen Mikes: Lettres de Turquie. Éd. sous la dir. de G. Tüskés, avant-propos d'A. Szerb, traduites de hongrois et annotées par K. Kaló et T. Fouilleul, avec des notes historiques de F. Tóth, éd. revue et préparée par M. Marty, Paris, Honoré Champion, 2011 (Bibliothèque d'études de l’Europe Centrale, Série « Littérature », 7). 
étaient parfois très différents de ceux de la diplomatie officielle. Durant la période qui nous intéresse, Louis XV concentra principalement son attention sur la Pologne où le parti francophile était assez fort. Son candidat français fut le prince de Conti qui était en correspondance secrète avec les ambassadeurs français à Varsovie, Constantinople, Stockholm et Saint-Pétersbourg, initiés, bien entendu, $\mathrm{au}$ « Secret du Roi ». L'enjeu de ces intrigues était le maintien d'un système d'alliance entre la France, la Turquie, la Pologne, la Suède et la Prusse afin de séparer l'Empire des Habsbourg d'avec la Russie. Un bon nombre d'agents hongrois au service de la France furent initiés au « Secret du Roi ». ${ }^{13}$

Dans sa lettre du 17 décembre 1726, Leszczynski fit une allusion à la mission d'André Tóth dont il transcrivit le nom phonétiquement à la hongroise: « Je vous felicite l'hereux retour de Totendrasz, et les Galliens chargé d'or qu'il a aporté vous estes plus heureux que le Roy d'Espagne. . . " ${ }^{14}$ Il s'agit là très probablement d'une mission de recrutement de troupes. Tóth devint plus tard l'un des meilleurs agents en Europe orientale. Lors de la seconde élection de Stanislas, comme l'a bien montré le regretté Gilles Veinstein dans son étude consacrée à ce sujet, Tóth joua un rôle important dans les négociations en Crimée, préparant ainsi le succès de Leszczynski. ${ }^{15}$ Initialement, Tóth arriva en mai 1733 à Constantinople où il essaya de trouver des recrues parmi les émigrés hongrois de Rodosto. L'ambassadeur de France à Constantinople, le marquis de Villeneuve, le retint d'autorité et modifia sa mission en l'envoyant comme son représentant auprès du khan des Tatars en Crimée. ${ }^{16}$ Tóth connaissait bien les membres les plus puissants de l'aristocratie polonaise et joua ainsi un rôle d'intermédiaire entre l'élite francophile polonaise, la diplomatie française, la Sublime Porte et leurs vassaux tatars. Il remplit cette fonction avec un tel succès que son fils, François, fut de son vivant désigné pour lui succéder dans la diplomatie orientale. Plus tard, François de Tott (1733-1793) contribua activement aux opérations diplomatiques françaises en faveur de la Pologne menacée par la politique de Catherine II. ${ }^{17}$

Finalement, le traité de Vienne régla la question de la succession de la Pologne d'une manière assez avantageuse pour les intéressés: Stanislas, battu en Pologne, gagna la Lorraine tandis que François III, duc de Lorraine, fut compensé

13 Jean Bérenger, Jean Meyer: La France dans le monde au XVIII ${ }^{\mathrm{e}}$ siècle. Paris 1993, pp. 66-67.

14 OSZKK, série Quart. Gall. 39 Lettres du roy de Pologne et d'autres princes fol. 12.

15 Gilles Veinstein: Les Tatars de Crimée et la seconde élection de Stanislas Leszczynski. In: Cahiers du monde russe et soviétique 11 (1970), pp. 24-92.

16 Lavender Cassels: The Struggle for the Ottoman Empire 1717-1740. London 1966, p. 90.

17 Voir sur la biographie de François de Tott: Ferenc Tóth: Un diplomate militaire français en Europe orientale à la fin de l'ancien régime. François de Tott (1733-1793). Istanbul 2011. 
par le grand-duché de Toscane. Stanislas s’installa donc à Nancy et à Lunéville, où il créa une petite cour qui fut fréquentée aussi par les gentilshommes hongrois résidant en France. Il nomma le comte Berchény, son ami de longue date, grand écuyer de sa cour le 21 avril $1738 .^{18}$ Cette dignité, hormis la direction du haras de Saaralbe et du personnel des écuries, avait une importance considérable dans les cérémonies auliques. En particulier, il devait proclamer le nouveau duc en tirant l'épée de la souveraineté de son fourreau. Ce fut le grand écuyer également qui portait les insignes royaux (les « quatre pièces de souveraineté »: la couronne, le sceptre, la main de justice et l'épée). Lors des entrées solennelles des duc dans leur capitale, le grand écuyer précédait le duc en portant l'épée ducale nue. ${ }^{19}$

Malgré l'amitié du beau-père de Louis XV, ou peut-être à cause de cela, la carrière de Ladislas Berchény ne fut pas facile à la cour de Versailles. Comme il se trouva souvent perdu dans le labyrinthe de la cour, il ne réussit jamais s'y intégrer complètement et se résigna à passer sa vie en seigneur hongrois dans le château de Luzancy. Beaucoup de familles hongroises s’implantèrent dans la vallée de la Marne, en Lorraine et en Alsace, bénéficiant ainsi de la proximité de la cour de Stanislas Leszczynski. Les projets politiques des deux émigrations favorisèrent la coopération entre Hongrois et Polonais. Même vingt ans après son arrivée en Lorraine, Stanislas Leszczynski ne cessa de fomenter des projets en Europe centrale. D’après le témoignage d'une lettre de Berchény adressée au comte d'Argenson, le roi Stanislas rêvait encore en 1756 de la reconstruction d'une alliance francophile en Europe centrale avec les Suédois, Polonais et Hongrois. ${ }^{20}$ Cependant la révolution diplomatique de 1756 bouleversa complètement les projets des émigrés hongrois, l'alliance franco-autrichienne devenant l'axe principal de la politique extérieure de la France.

Dans cette nouvelle situation, la fraternité hungaro-polonaise ne cessa point. Les bonnes relations entre le comte Berchény et Stanislas Leszczynski facilitèrent la carrière des descendants du comte. Notons ici le mariage de François Nicolas de Berchény, fils du maréchal, qui fut contracté en 1757 avec Agnès Berthelot de Baye dont le père, le baron de Baye, était très attaché à l'ancien roi de Pologne. Il fut maréchal de camps et armées de Lorraine. La demoiselle de Baye apporta une grosse fortune (une dot de 100000 livres) à son époux, ce dont même le duc de Luynes nous laissa un témoignage dans ses mémoires: « M. de Berchiny,

\footnotetext{
18 Zachar (note 3), p. 126.

19 Henri Lepage: Les offices des duchés de Lorraine et de Bar et la Maison des ducs de Lorraine. In: Mémoires de la Société d'archéologie lorraine, seconde série, XI vol. (1869) pp. 370-373.

20 Ferenc Tóth: Magyar vonatkozású dokumentumok a d’Argenson család levéltárában I.[Documents relatifs aux Hongrois dans les archives de la famille d'Argenson I.] In: Hadtörténelmi Közlemények 123 (2010), p. 907.
} 


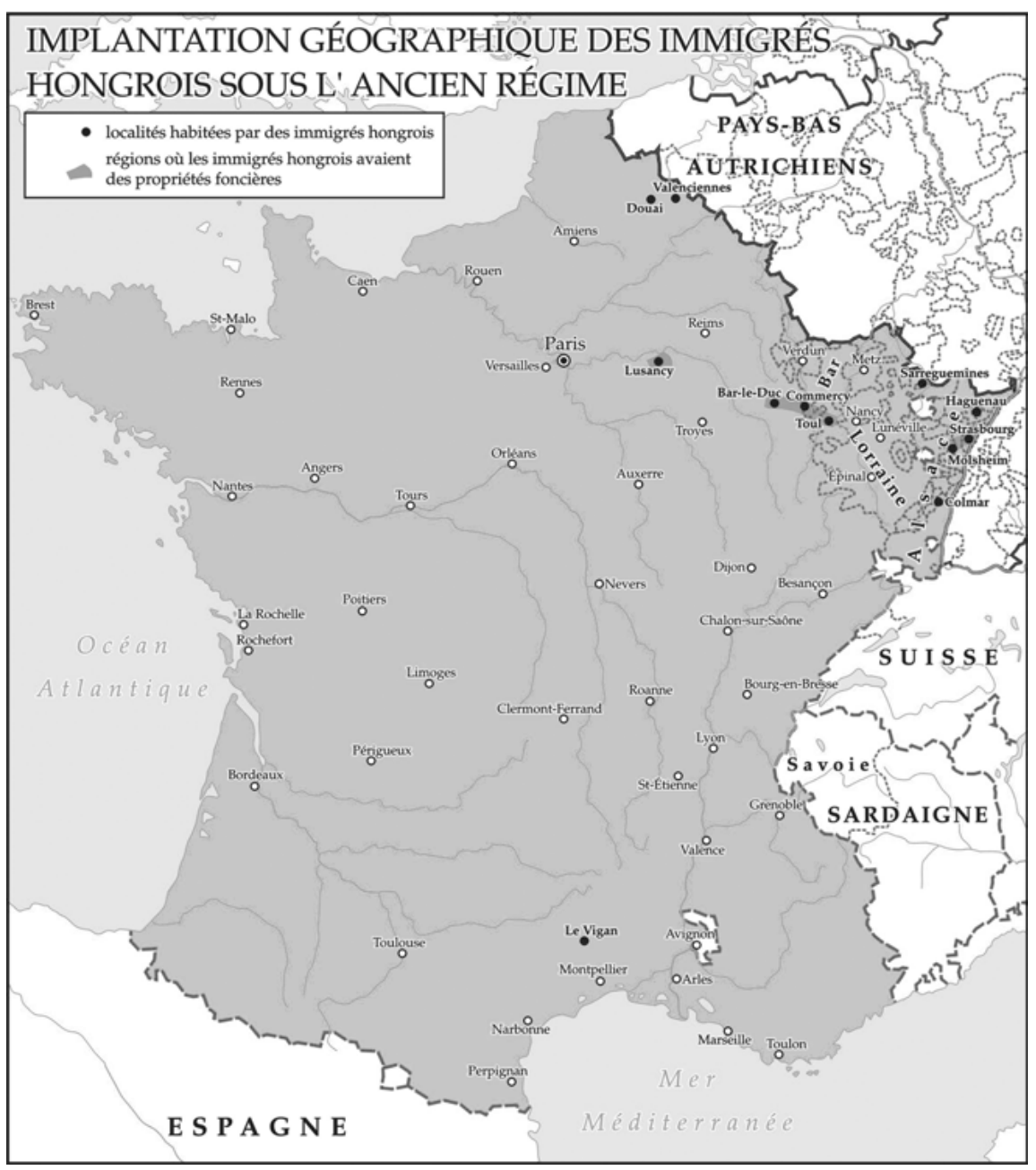

lieutenant général, marie son fils avec la fille de M. de Bail, commandant des cadets du roi de Pologne, duc de Lorraine. Le roi de Pologne donne une place de chambellan au mari et 4000 livres d'appointements. Le Roi assure 4000 livres de douaire. ${ }^{21}$

21 Mémoires du duc de Luynes sur la Cour de Louis XV (1735-1758). Tome XVI. Paris 1864, p. 20. 
La bienveillance de la reine Marie Leszczynska, fille de Stanislas, joua un rôle primordial dans l'avancement du maréchal Berchény et des membres de sa famille. Avant d'être nommé maréchal de France, Berchény sollicita le concours de la reine pour recevoir la plaque de grand-croix de l'ordre de Saint-Louis et un gouvernement militaire. Au moment de la libération d'une place dans l'ordre au printemps 1753, Berchény remit une supplique à la reine par l'intermédiaire de son confesseur polonais, le père Radominski, qu'il avait connu à Lunéville: « Employez votre protection pour me faire obtenir la grand-croix de Saint-Louis qui vaque. » Au début de l'automne de la même année, il remercia la reine ainsi: «J'ose espérer la continuation de ses bontés et de sa protection, mon ambition étant de vous servir toujours, Madame, et le roi votre père. ${ }^{22} \mathrm{La}$ reine intervint sans doute en faveur de Berchény dans le processus de sa nomination de maréchal de France. Elle ne cacha point sa satisfaction en félicitant le comte peu après l'événement: « Je suis ravie Mon Cher Marechal de Vous nommer ainsi, je ne Vous demande pour reconnoissance du Desir que j'en ay Eû, que d'augmenter s'il se peut, d'attachement pour mon Papa; mettez moy à ses pieds, Et soyez sûr mon cher Berchénÿ, que je Vous aime et Estime de tout mon cœur. Dites a La Marechale ma joie. ${ }^{23}$

L'apparition des Hongrois fut donc liée aux relations cordiales entre le roi Stanislas et Ladislas Berchény. On peut retrouver autour du château de Lusancy les traces des petites communautés hongroises et polonaises composées des officiers du régiment de hussards Berchény. Dans les actes de baptêmes des paroisses des environs, on trouve souvent des parrainages entre Hongrois et Polonais (Tott, Benyo ou Benyowski, Duksa etc.) ce qui prouve les liens étroits entre eux. Les recherches récentes d'histoire locale sur le manoir fortifié de Chamigny, nommé Rougebourse et qui servait de domicile à plusieurs familles d'officiers hongroises et polonaises, ne font que renforcer l'existence des liens entre les deux communautés émigrées. ${ }^{24}$ Toutefois, nous pouvons repérer d'autres personnages non moins intéressants. Le comte Valentin Joseph Esterhazy fut également très lié au cercle polonais de Stanislas. Son père, Antal Esterhazy, résida également en Pologne avant de rejoindre le prince Rákóczi en Turquie où il termina sa vie. Son fils fut enrôlé à Rodosto en 1720 pour le régiment Berchény. Plus tard, il fonda à Strasbourg, en 1735, la troisième unité à majorité hongroise, le régiment Esterhazy. Après sa mort survenue en 1743, son fils Ladislas Valentin Esterhazy se rendit avec sa mère

22 Cité par Anne Muratori-Philip: Marie Leszczynska. Épouse de Louis XV. Paris 2010, p. 229.

23 OSZKK, série Quart. Gall. 39 Lettres du roy de Pologne et d'autres princes fol. 139.

24 Information cordialement fournie par M. A. Bouteveille. 
et sa sœur à Versailles pour implorer l'aide de la reine, ce dont il nous laissa des témoignages dans ses Mémoires: « Le seul secours qu'elle put obtenir fut une petite pension sur la cassette du roi, trop faible pour subvenir aux frais de l'éducation de ses enfants. Elle recourut alors à la reine, fille du roi Stanislas. La reine s'intéresse à un nom qu'elle avait connu en Pologne. Elle voulut que ma mère nous menât chez elle, ma sœur et moi. Elle fit entrer ma sœur à Saint-Cyr et me destina une place dans ses pages, quand je serais d'âge. ${ }^{25}$ Plus tard, le jeune Esterhazy fut élevé par le comte Berchény et bénéficia également du rayonnement de la cour de Stanislas. ${ }^{26}$

Parmi les Hongrois les plus initiés à la cour de Lunéville, il convient d'insister sur l'importance de l'intendant Joseph Jankovich de Jeszenice (1706-1768) dont le père participa très probablement à la guerre d'indépendance du prince Rákóczi. Le jeune Jankovich entra vers 1722 au service du comte François-Maximilien Ossolinski, qui lui confia la surveillance de son domaine de Prusse. Quand Stanislas Leszczynski revint en France en mai 1736, Jankovich fut chargé d'acheminer vers la Lorraine, avec des meubles et la cave de son maître, différents effets du roi et de guider les serviteurs. Dans la tradition familiale des Jankovich, il fut souvent question du transfert des « joyaux de la couronne » et de « missions diplomatiques de la plus haute importance ». En 1759, il épousa la comtesse Anna Krotunszka qui était une filleule de Leszczynski et protégée du duc d'Ossolinski. ${ }^{27}$ Jankovich devint alors contrôleur de la maison du roi. Le couple demeura à Lunéville et, un an avant la mort de Stanislas Leszczynski, ils achetèrent la maison du maître-pâtissier de Lunéville. ${ }^{28}$ Leur fils AntoineStanislas-Nicolas-Pierre-Fourier, né à Lunéville en 1763 et qui eut le roi Stanislas comme parrain, fit une belle carrière en France. En 1792, il épousa la petite-fille du sculpteur Étienne Falconet, il fut créé baron héréditaire en 1820, président du collège électoral de Château-Salins, il fut de 1806 à 1830 conseiller général, en 1815 et sans interruption de 1820 à 1830 député de la Meurthe. A la

25 Mémoires du comte Valentin Esterhazy. Paris 1905, pp. 8-9.

26 Ibid., pp. 16-28.

27 Pierre Boyé: La Cour Polonaise de Lunéville (1737-1766). Nancy, Paris, Strasbourg 1926, pp. 143-144. Cf. Notice biographique et généalogique sur M. le baron de Jankovitz de Jezenice (Extrait de la Biographie des membres de la Chambre des députés, par M. de Lansac). Paris 1847; József Pozsonyi: The History of the Jankovich de Jeszenice Family. Debrecen 2014, p. $28-30$.

28 Stéphane Gaber: L'entourage polonais de Stanislas Leszczynski à Lunéville 1737-1766. Thèse de doctorat, Université de Nancy 1972, p. 67. 
fin de sa vie il laissa une belle collection de correspondance de Stanislas Leszczynski à la Bibliothèque Nationale Széchényi de Budapest. ${ }^{29}$

On note dans les sources la présence à la cour de Stanislas à Lunéville d'un certain comte Antoine Eperjessy, Hongrois naturalisé polonais qui y séjourna en 1739. Pendant la guerre de succession de Pologne, en 1733-34, il soutint militairement le parti de Leszczynski en Pologne, ce qui lui valut le grand respect et l'amitié du roi. Lors de sa visite à Lunéville, Leszczynski le récompensa richement, comme en témoigne la Gazette de Hollande: « Il alla d'abord rendre ses respects au roi, qui le reçut avec de grandes marques de bonté, et Sa Majesté voulant reconnaître son zèle pour son service lui a fait plusieurs magnifiques présents, et lui a donné entre autres une tabatière d'or de grand prix et ornée de son portrait. Le comte est parti depuis pour retourner en Pologne, entièrement satisfait du bon accueil qui lui a été fait en cette cour. $»^{30}$

Parmi les membres de la famille Benyovszky, on connaît surtout le comte Maurice-Auguste Benyovszky qui naquit en 1745 à Verbó en Hongrie et qui, après avoir participé à la Confédération de Bar, fit une carrière pleine d'aventures dans le monde et se distingua dans la colonisation de Madagascar. Il convient de rappeler que sa famille s'enracina bien avant en Normandie et plus tard dans la vallée de la Marne, près du château de Lusancy. Il n'est pas surprenant que, lorsque Maurice-Auguste Benyowsky, arriva en France en 1772, il y trouva un très bon accueil auprès de son oncle qui était commandant du château de Bar-le-Duc. ${ }^{31}$

29 Voici un extrait de la lettre de donation de Stanislas Jankovich de Jeszenicze: « En déposant au Musée National de Pesth, des Lettres autographes de plusieurs souverains et princesses restées entre les mains de mon Pere, par suite de l'auguste confiance qu'avait en lui Sa Majesté Stanislas premier Roi de Pologne; Je crois offrir à l'illustre Patrie de mes ancêtres, le plus noble hommage qui soit en mon pouvoir. Privé d'un fils unique qui faisait mon bonheur, ma gloire, l'espoir de voir notre nom honnorablement soutenu par lui, je saisis ce moyen de faire subsister en Hongrie quelques traces de notre existance d'un siècle en France. » OSZKK, série Fol. Gall. 1 Lettre de Stanislas Jankovich de Jeszenicze.

30 Cité par St. Gaber (note 28), p. 135.

31 Il nous raconte ainsi, dans ses mémoires, le début de son séjour en France: « Le 8 d'août, j'arrivai en Champagne, où était alors le ministre, qui me reçut avec distinction et cordialité, et qui me proposa d'entrer au service de son maître, avec l'offre d'un régiment d'infanterie; ce que j'acceptai, à condition qu'il plairoit à Sa Majesté de m'employer à former des établissements au-delà du Cap. J'eus aussi le bonheur de trouver en France mon oncle, le comte de Benyow, commandant de la ville et du château de Bar, commandeur de l'ordre royal de SaintLazare, et chevalier de Saint-Louis. Les secours de ce digne parent, et la bienveillance de Sa Majesté, me mirent en état d'envoyer un exprès en Hongrie, pour chercher mon épouse et mon fils. » Voyages et mémoires de Maurice-Auguste, comte de Benyowszky. Paris 1791, p. 209. Les documents conservés aux Archives Nationales (S. O. M. Fonds Madagascar C5 A3 nº 74) et aux 
Les Pollereczky faisaient partie également des familles émigrées hungaropolonaises qui bénéficièrent de l'appui de l'entourage hongrois de Stanislas Leszczynski. Plusieurs membres de la famille furent nommés commandant de régiments de hussards et, plus tard, se distinguèrent durant la guerre d'indépendance américaine. ${ }^{32}$

\section{La Société royale des sciences et belles-lettres de Nancy et les Hongrois}

L'actuelle Académie de Stanislas fut fondée à Nancy le 28 décembre 1750 par Stanislas Leszczynski sous le nom de Société royale des sciences et belles-lettres de Nancy. Elle fut créée en même temps que la bibliothèque publique à laquelle elle était étroitement liée. Un des buts de la Société royale consistait en la sélection des ouvrages de la bibliothèque. De même, le roi institua deux prix, l'un pour les sciences et l'autre pour la littérature et les arts, qui seraient distribués à des sujets lorrains. Le premier noyau académique regroupait le bibliothécaire et quatre membres à vie qu'on appelait les censeurs des ouvrages. Le premier bibliothécaire était un ancien secrétaire et homme de confiance du roi de Pologne, Pierre-Joseph de Solignac, qui s'était attaché à Stanislas en Pologne et avait partagé les dangers du siège de Dantzig avec son maître. Il était déjà membre de plusieurs académies dont l'Académie des inscriptions et belles-lettres de Paris. ${ }^{33}$ L'idée initiale était de créer une véritable académie à Nancy. Ce projet était à la fois philosophique, scientifique et politique. La Lorraine ayant une position géographique centrale, le projet avait des enjeux géopolitiques européens. C'était un noyau de pôle intellectuel européen entre Paris et Berlin et entre Rome et Londres. Pour reprendre les mots de Jacques Vier, il s'agissait d'une " plate-forme stratégique » ${ }^{34}$ au niveau géographique ainsi que sur le plan des idées. Ayant des rapports très étroits avec la Pologne,

Archives Départementales de Meuse (ADM, série E dépôt, BB 43 fol. 58. et BB 44 fol. 48.) confirment également qu'à cette période, le comte Paul de Benyo (sic!), ancien capitaine de régiment de hussards Berchény, était commandant du château de Bar.

32 Voir à ce sujet: Ferenc Tóth: Fraternité dans l'émigration: nobles hongrois et polonais en France au XVIII ${ }^{\mathrm{e}}$ siècle. In: Jaroslaw Dumanowski, Michel Figeac (éd.): Noblesse française et noblesse polonaise. Mémoire, identité, culture XVI ${ }^{\mathrm{e}}-\mathrm{XX}^{\mathrm{e}}$ siècles. Bordeaux 2006, pp. 75-87.

33 Christian Pfister: Histoire de l'Académie de Stanislas, in Jean Favier (éd.): Table alphabétique des publications de l'Académie de Stanislas (1750-1900). Nancy 1902, pp. 2-3.

34 Jacques Vier: L'activité d'une académie provinciale au XVIII ${ }^{\mathrm{e}}$ siècle. L'Académie de Stanislas à 1766. In: Revue d'Histoire littéraire de la France (1926), p. 348. 
cette institution avait pour vocation de créer un pont entre l'Europe de l'Ouest et l'Europe centrale et orientale. ${ }^{35}$

Ce pont existait bel et bien entre la Pologne et la Lorraine où la cour de Stanislas était véritablement une plaque-tournante d'intellectuels. L'évêque Joseph André Zaluski (1702-1774) fut certainement un exemple frappant des personnages qui entretenaient les relations entre les deux pays. Zaluski était un ecclésiastique, un mécène et un bibliophile très connu de son temps. Pendant son séjour lorrain (1737?-1742), il fut grand aumônier de Stanislas Leszczynski et joua un rôle de médiation important à travers sa correspondance étendue avec des savants, hommes de lettres et érudits en Europe. Dès 1732, il forma un projet ambitieux de créer une grande bibliothèque publique, une bibliographie de la littérature polonaise, et une académie des sciences avec des projets de publications. Il consacra sa vie, avec son frère André Stanislas Zaluski, à ce vaste projet (Programma litterarium) et joua un rôle d'intermédiaire intellectuel entre Nancy et la Pologne. ${ }^{36}$ Il commença à collectionner les ouvrages pendant son séjour lorrain et en laissa à son départ une partie au roi de Pologne, le " Petit fonds Zaluski ", ${ }^{37}$ qui devint par la suite l'une des bases de la bibliothèque publique fondée par Stanislas en 1750. En Pologne, il fonda la fameuse Bibliothèque Zaluski (Bibliotheca Zalusciana) à Varsovie, première bibliothèque publique polonaise et l'une des premières en Europe, et qui devint bientôt la plus grande bibliothèque polonaise avec une collection prestigieuse de livres, manuscrits, cartes, estampes etc. Vers la fin des années 1780 , cette bibliothèque abritait environ 400000 documents. ${ }^{38}$

Outre les émigrés hongrois, leurs compatriotes voyageurs étaient aussi les bienvenus dans la cour du roi Stanislas. Le comte Joseph Teleki passa en mars 1761 un séjour très agréable en Lorraine, où il fut très bien accueilli par les Berchény ainsi que par l'ancien roi de Pologne. Il en rendit un témoignage intéressant dans son journal où il décrivit non seulement les monuments intéressants à voir à Nancy et à Lunéville, mais nous laissa également un témoignage de la

35 Doyen Laurent Versini: Pourquoi Stanislas a-t-il fondé une Académie ? In: Stanislas et son Académie (note 1), p. 32.

36 Stanislaw Roszak: La correspondance de Józef Andrzej Zaluski: du « Cercle des correspondants » à l'egodocument dans les recherches sur la culture polonaise. In: François Cadilhon, Michel Figeac, Caroline Le Mao (éd.): La Correspondance et la construction des identités en Europe Centrale (1648-1848). Paris 2013, pp. 276-278.

37 Pierre Boyé: Le petit fonds Zaluski de la bibliothèque publique à Nancy. In: Bulletin de la Société d'archéologie lorraine (1920), pp. 112-119. Cf. Stéphane Gaber: Le fonds Zaluski de la bibliothèque publique de Nancy. In: Annales de l’Est (1975), pp. 157-165.

38 Stéphane Gaber: Un bibliophile polonais à la cour de Stanislas: Joseph-André Zaluski. In: Le Pays lorrain (1974), pp. 65-82. 
vie quotidienne du vieux Stanislas. Il décrivit celui-ci comme un homme âgé bienveillant et intelligent. Il fut très intéressé par la cour du roi de Pologne, surtout par les gardes du corps du roi et les travaux du château de Lunéville où il passa plusieurs jours. Grâce à la recommandation du vieux Berchény, Teleki assista à plusieurs reprises aux repas à la table du roi, dont il put observer le caractère et les habitudes. Il put décrire également le milieu hongrois des Berchény, qu'il qualifia de patriote hongrois conservant une langue et une culture hongroises bien vivantes malgré son exil de presque cinquante ans ! ${ }^{39}$

Les bonnes relations entre Stanislas Leszczynski et Ladislas Berchény se conservèrent au fur et à mesure de l'avancement de leur âge. Le château de Berchény à Luzancy, dans la vallée de la Marne, servit souvent d'escale à Stanislas lorsqu'il se rendait à Versailles. Pratiquement le seul témoignage conservé de cette époque est une plaque de cuivre buriné posée sur le mur de l'escalier du château rappelant les visites royales: «En 1765 le 19 du mois de septembre Marie Leczinska Reine de France en revenant de voir son père le Roy de Pologne à Commercy se détourna exprès de La Ferté sous Jouarre pour venir à Lusancy dîner chez le Maréchal de Bercheny pour qui elle a eu ainsi que pour sa famille des bontés distinguées. Stanislas $\mathrm{I}^{\mathrm{er}}$ Roy de Pologne Duc de Lorraine et de Bar allant tous les ans voir sa fille la Reine à Versailles dinoit et couchoit à Lusancy à son passage et à son retour. " ${ }^{40}$

Nous avons d'autres informations qui confirment l'existence de relations entre les personnages hauts en couleurs de la Société royale et ceux de l'émigration hongroise en Lorraine. Notons ici les deux secrétaires de Stanislas, Pierre Joseph de La Pimpie, chevalier de Solignac et Jean-Pierre Tercier, qui avaient partagé le sort des partisans du roi de Pologne après la chute de Dantzig et participèrent activement à la vie académique et intellectuelle lorraine. Hormis le réseau académique, ces personnages, surtout le premier commis Tercier, étaient initiés à la diplomatie secrète de Louis XV, le fameux Secret du Roi, qui préparait des projets à contre-courant de la diplomatie officielle française en Europe centrale et orientale. ${ }^{41}$ Les Tott père et fils, diplomates, y étaient employés activement et nous pouvons présumer que les Berchény en firent parties. Il faut encore

39 Egy erdélyi gróf a felvilágosult Európában (Teleki József utazásai 1759-1761) [Un comte transylvain dans l'Europe éclairée (Les voyages de Joseph Teleki 1759-1761)]. Éd. par Gábor Tolnai. Budapest 1987, pp. 219-224. Cf. Gabriel Tolnai: La Cour de Louis XV. Journal de voyage du comte Joseph Teleki. Paris 1941.

40 Cité par Alix Bouteveille: Luzancy. Un château au fil du temps, Histoire, énigmes et controverses, seigneurs et châtelains. Coulommiers s. d., p. 51.

41 Voir sur ce sujet: Jean-Fred Warlin: J.-P. Tercier: l'éminence grise de Louis XV. Un conseiller de l'ombre au Siècle des lumières. Paris 2014. 
souligner un troisième réseau qui constituait un creuset pour l'élite cosmopolite autour de la cour de Stanislas: les loges maçonniques, auxquelles l'élite hongroise adhérait massivement. ${ }^{42}$

Ladislas Berchény avait déjà reçu une formation soignée au sein de la Compagnie Nobiliaire, la garde du corps du prince Rákóczi qui était une unité élite de l'armée hongroise durant la guerre d’indépendance. Créée en 1707 par le prince, elle était composée de cent jeunes cavaliers nobles hongrois. En dehors de la surveillance permanente du prince, ils recevaient une éducation distinguée, ce qui rapprochait cette unité des académies équestres de l'époque. Dans la vie du jeune László Berchény, le service dans la Compagnie Nobiliaire fut un élément décisif. Le jeune Berchény commença également l'étude du français en Hongrie, en tant que membre de la Compagnie Nobiliaire du prince Rákóczi. ${ }^{43}$ Plus tard, comme propriétaire de régiment, il emprunta beaucoup à la Compagnie Nobiliaire. Par exemple, l'uniforme du régiment Berchény était une copie fidèle de celle que les gardes du corps du prince avaient porté naguère. Il est intéressant de noter que Marie-Thérèse recourut aussi à la même source, la Compagnie Nobiliaire de Rákóczi, lorsqu'elle fonda la garde du corps nobiliaire hongroise à Vienne. ${ }^{44}$

Même si nous ne trouvons pas beaucoup de traces de la participation des Hongrois aux activités de l'Académie de Stanislas, un ouvrage dédié au comte Ladislas Berchény parmi les publications issues de cette société savante mérite bien notre attention. Il s'agit d'un petit livre intitulé Le politique vertueux. La candeur et la bonne foi sont plus nécessaires à l'Homme d'Etat, que la ruse et la dissimulation, publié à Nancy en 1762 chez l'imprimeur Jean-Baptiste Hyacinthe Leclerc. L'auteur du livre s'appelait François-Hubert Aubert, et fut avocat de la cour et des conseils de Stanislas à Lunéville. L'ouvrage porte sur son frontispice un beau portrait en buste du maréchal Berchény, en habit militaire et décoré $\mathrm{du}$ cordon bleu, réalisé très probablement par le graveur Dominique Collin. ${ }^{45}$ Ce qui est surprenant dans l'organisation du texte du livre réside dans la

42 Ferenc Tóth: Ascension sociale et identité nationale. Intégration de l'immigration hongroise dans la société française au cours du XVIII ${ }^{\mathrm{e}}$ siècle (1692-1815). Budapest 2000, pp. 152-154.

43 Zachar (note 3), pp. 49-60.

44 De façon assez paradoxale, tandis que les gentilshommes hongrois de Vienne devenaient les propagateurs zélés des idées des Lumières (György Bessenyei, Ábrahám Barcsay etc.), leurs compatriotes émigrés en France prenaient une position plutôt conservatrice et ils restèrent les défenseurs fidèles de la Monarchie sous l'Ancien Régime et pendant la Révolution. Voir sur la garde nobiliaire hongroise: Aladár Ballagi: A magyar királyi testőrség története [Histoire de la garde du corps royale hongroise]. Pest 1872.

45 M. Beaupré: Deuxième supplément à la notice sur Dominique Collin et Yves-Dominique Collin. In: Mémoires de la Société d'archéologie lorraine. Nancy 1866, p. 164. 
grandeur et la richesse de la dédicace. Elle comprend soixante-six pages et contient une biographie très détaillée du comte de Berchény. Malgré les inexactitudes orthographiques concernant les noms propres hongrois, l'auteur devait avoir des renseignements très précis sur l'histoire hongroise et sur la famille du comte. Son père, Nicolas Berchény, ancien général de l'armée du prince Rákóczi, y est également bien présenté ainsi que beaucoup de détails de l'histoire de la fin du XVII ${ }^{\mathrm{e}}$ siècle, comme l'histoire du tribunal d'Eperjes qui provoqua beaucoup de mécontentement en Hongrie. Néanmoins, la jeunesse du comte au cours de la guerre d'indépendance hongroise est complètement passée sous silence, et ce peut-être à cause des tentatives de retour du comte en Hongrie vers la fin de sa vie. Son service militaire en France y est décrit avec ses hauts faits et ses actions d'éclats durant les guerres de succession de Pologne et d'Autriche et de la guerre de Sept Ans. Les mariages et les enfants du comte y sont également bien représentés. L'éloge du comte se termine par un véritable hymne à ses vertus, comparées aux héros classiques et modernes: « M. le Maréchal de Bercheny, dit-on, dans toutes les sociétés, est ami comme Ephestion l'étoit, époux comme Caton, sage comme Socrate, pere comme Paul Emile, brave comme le vainqueur d'Annibal, prudent \& citoyen comme Fabius, homme de bien comme Scipion Nasica, \& vertueux comme Sully. " ${ }^{46}$

Le comte Berchény imita les initiatives de Stanislas et créa lui-même une bibliothèque riche, malheureusement dispersée durant la révolution française mais dont le catalogue témoigne toujours d'un vif intérêt culturel et littéraire de la noblesse hongroise exilée. Leur production littéraire était tout à fait intéressante. Notons ici l'importance des mémorialistes francophones hongrois qui, dans la lignée du prince Rákóczi, nous laissèrent des témoignages extraordinaires. Si l'on fait abstraction des Mémoires disparues du comte Berchény dont encore à la fin du XVIII ${ }^{\mathrm{e}}$ siècle l'éditeur hongrois des Lettres de Turquie de Clément Mikes ${ }^{47}$ se servit utilement, nous avons trois mémoires plus ou moins liés au milieu intellectuel et éclairé lorrain: ceux du comte Valentin Esterhazy, fils adoptif du comte Berchény, ceux du baron de Tott, fils d'un ancien diplomate au service des projets polonais du roi de France et ceux plus controversés de Maurice-Auguste Benyovszky, neveu du commandant de Bar-le-Duc. Le goût de la littérature s'infiltra même dans les rangs des officiers de hussards moins connus. D’après une note des archives militaires de Vincennes, le vétéran hongrois Charles-Michel -Jean Szilágyi d’Horogszeg, retiré à Mirecourt, au sud de Nancy, commença à

46 François-Hubert Aubert: Le politique vertueux. La candeur et la bonne foi sont plus nécessaires à l'Homme d'Etat, que la ruse et la dissimulation. Nancy 1762, pp. LXV-LXI.

47 Voir sur cet ouvrage sa récente édition critique francophone: Mikes (note 12). 
traduire, pour son amusement, les œuvres de Polybe en hongrois ! ${ }^{48}$ En cherchant un peu dans les familles hongroises implantées en Lorraine, nous pouvons même trouver un poète en la personne de Ladislas Lancelot Dessoffy, chanoine de Toul, qui se distingua comme auteur d'oraisons funèbres de la cour de Vienne durant son émigration. ${ }^{49}$

La cour de Stanislas Leszczinsky à Lunéville accueillait beaucoup d'hôtes illustres. Le philosophe Voltaire y séjournait aussi à l'époque où le comte Ladislas Berchény fut nommé gouverneur de Commercy par le roi Stanislas (1748). ${ }^{50}$ Un foyer similaire qui abritait encore beaucoup de Hongrois fut le château de Berchény à Luzancy. Le maréchal était un bon maître qui laissa à son fils un domaine considérable. François Antoine de Berchény continua l'œuvre de son père et son château fut célèbre parmi les gentilhommières situées à proximité de Paris. Le marquis de Bombelles le voyait ainsi durant son voyage à Luzancy en 1782: « J'avais promis à mon ami le comte de Bercheny d'aller le voir à sa terre de Lusancy [...] Cet homme intéressant n'a jamais eu un écart. Bon fils, bon frère, bon mari, il n'est pas moins tendre père, il n'est pas moins bon seigneur. Ses paysans l'adorent par son équité et sa sensible humanité. Ses paysannes en raffolent par son soin pour ramener, pour inventer autour de lui tous les plaisirs champêtres. ^ ${ }^{51}$

Le château des Berchény à Lusancy avait également une belle collection de peintures et une bibliothèque dont il ne nous reste que la liste des ouvrages vendus aux enchères des 8 et 9 Floréal de l'an III de la République. Le montant de la vente des toiles s'éleva à deux mille quatre cent cinquante et un francs. Le comte Berchény, selon le témoignage du mémorialiste Ladislas Valentin Esterhazy, commença sa formation à un âge avancé: « Le comte, depuis maréchal de Bercheny, était un parfait honnête homme de l'ancien temps. Il avait commencé à s'instruire à l'âge où les autres hommes oublient ce qu'ils ont appris. " ${ }^{52}$

Le maréchal de Berchény, et plus tard ses enfants, réunirent une collection remarquable de livres imprimés et de manuscrits. Une partie de cette collection fut inventoriée lors de la confiscation des biens de la maison de Berchény. La liste des ouvrages, dressée au moment de la confiscation des biens du comte François Antoine Berchény, émigré en Autriche, nous permet d'évaluer

48 Raimond Boissau: Dictionnaire des officiers de hussards de l'Ancien Régime. Des origines à Valmy (1693-1792). Paris 2015, p. 186.

49 Tóth (note 42), pp. 229-230.

50 Voir à ce sujet: Gaston Maugras: La Cour de Lunéville au XVIII ${ }^{\mathrm{e}}$ siècle. Paris 1925, p. 165.

51 Journal du marquis de Bombelles. Tome I. Éd. par Frans Durif, Jean Grassion. Genève 1978, p. 120.

52 Mémoires du comte Esterhazy (note 25), p. 12. 
l'importance de la bibliothèque des Berchény. La partie de cette bibliothèque qui survécut aux troubles de la Révolution constitue encore, avec ses neuf cent quarante-deux volumes, un ensemble magnifique même par rapport aux grandes bibliothèques nobiliaires et parlementaires de l'époque. ${ }^{53}$

Voici le tableau représentant la répartition thématique des livres ${ }^{54}$ :

\begin{tabular}{lrr}
\hline Catégorie & Nombre & Pourcentage \\
\hline Histoire & 406 & $43,1 \%$ \\
Belles-lettres & 141 & $15,0 \%$ \\
Sciences et arts & 201 & $21,3 \%$ \\
Philosophie & 38 & $4,0 \%$ \\
Religion & 21 & $2,2 \%$ \\
Droit & 68 & $7,2 \%$ \\
Autres & 68 & $7,2 \%$ \\
Total & 943 & $100 \%$ \\
\hline
\end{tabular}

Nous pouvons donc constater que les ouvrages historiques et scientifiques, surtout les manuels concernant l'art militaire, dominaient la bibliothèque des Berchény. On y trouve aussi à côté des livres des auteurs anciens, ceux des penseurs les plus connus des Lumières tels que Voltaire, Rousseau et Montesquieu. Naturellement, les livres des membres éminents de l'émigration hongroise figurent de même dans la collection des Berchény, notamment le Testament politique et moral du prince Rákóczi, l'Histoire des révolutions de Hongrie de l'abbé Brenner, Le Partisan de Jeney ainsi que les Mémoires sur les Turcs et les Tartares du baron de Tott. Par ailleurs, nous avons une liste similaire de la bibliothèque, ensuite vendue, du baron de Tott, qui témoigne également d'une forte influence des idées des Lumières dont il fut lui-même aussi un porte-parole dans ses Mémoires. ${ }^{55}$

53 Michel Marion: Les bibliothèques privées à Paris au milieu du XVIII ${ }^{\mathrm{e}}$ siècle. Paris 1978, pp. 176-184.

54 D’après Forster (note 9), pp. 91-115.

55 Ferenc Tóth: Ex libris baron de Tott. Portyázás egy XVIII. századi magyar származású francia huszártiszt könyvtárában [Ex libris baron de Tott. Voyage dans la bibliothèque d'un officier de hussards français d'origine hongroise au XVIII ${ }^{\mathrm{e}}$ siècle]. In: Hadtörténelmi Közlemények 119 (2006), pp. 389-414. 
$\mathrm{Au}$ terme de cette présentation, il convient de rappeler que les gentilshommes hongrois vivant dans les environs de Nancy et de la cour de Stanislas pouvaient bénéficier du rayonnement de ces centres culturels, scientifiques et artistiques. Liens locaux et étrangers semblent s'être conjugués pour faire de Nancy une plaque tournante intellectuelle en Lorraine et de l'Est de la France. Les Polonais, Allemands, Italiens et Hongrois y contribuaient et, en même temps, bénéficiaient des influences des différentes institutions nouvellement créées. Malgré les différentes influences et les relations personnelles étroites, remarquons néanmoins une différence entre le statut des officiers de hussards hongrois et les savants des académies scientifiques et artistiques. En effet, il s'agit de deux structures académiques différentes qui commençaient à se consolider à cette période: les académies équestres et les académies des sciences et des arts. Dans l'Encyclopédie de Diderot et de d'Alembert, les membres sont distingués par deux termes différents: les académistes et les académiciens. L'auteur de leur entrée commune explique ainsi cette différence: «Ils sont l'un \& l'autre membres d'une société qui porte le nom d'Académie, \& qui a pour objet des matieres qui demandent de l'étude \& de l'application. Mais les sciences \& le bel esprit font le partage de l'Académicien, \& les exercices du corps occupent l'Académiste. L'un travaille \& compose des ouvrages pour l'avancement \& la perfection de la littérature: l'autre acquiert des talens purement personnels. " ${ }^{56}$ Dans le cas de nombreux nobles hongrois implantés à proximité de Nancy cette différence semblait s'effacer grâce à l'influence des œuvres de Stanislas Leszczynski.

56 Encyclopédie ou dictionnaire raisonné des sciences, des arts et des métiers par une Société de gens de lettres; mis en ordre et publié par M. Diderot, et quant à la partie mathématique, par M. d'Alembert. Tome I. Genève 1777, p. 222. 